\title{
Cartographie des interstices vocaux. De la parole et de l'écoute au théâtre
}

The vocal interstices' mapmaking. About speech and listening in the theatre

\section{Daniel Deshays}

\section{(2) OpenEdition \\ 12 Journals}

Édition électronique

URL : http://journals.openedition.org/rsl/1082

DOI : $10.4000 /$ rsl. 1082

ISSN : 2271-6246

Éditeur

Éditions Rue d'Ulm

Référence électronique

Daniel Deshays, "Cartographie des interstices vocaux. De la parole et de l'écoute au théâtre », Revue Sciences/Lettres [En ligne], 5 | 2017, mis en ligne le 02 octobre 2017, consulté le 20 avril 2019. URL http://journals.openedition.org/rsl/1082 ; DOI : 10.4000/rsl.1082

Ce document a été généré automatiquement le 20 avril 2019

(c) Revue Sciences/Lettres 


\title{
Cartographie des interstices vocaux. De la parole et de l'écoute au théâtre
}

The vocal interstices' mapmaking. About speech and listening in the theatre

\author{
Daniel Deshays
}

1 Si la voix demeure l'élément phare du théâtre, elle n'est qu'un des multiples éléments sonores scéniques. Je voudrais ici la replacer dans la globalité sonore du théâtre y compris face aux silences. Le mot énoncé tient un équilibre avec les silences qui l'entourent, des silences respiratoires. Les autres éléments sonores issus des actions de jeu ou introduits par l'entremise de haut-parleurs ne sont pas moins importants. Cette reconsidération globale veut rappeler que le son ne peut être pensé dans la séparation vers laquelle l'écoute $d u$ sens nous conduit pourtant implicitement, vers une voix considérée seule dans l'entre-soi établi avec l'écriture dramatique. La voix est du son et du sens, et le sens dépend du son, nous disait Ferdinand de Saussure ${ }^{1}$.

\section{Le travail du son et le silence}

2 Le recul me permet à présent d'apercevoir ce qu'il m'était impossible d'atteindre dans l'urgence où nous tient chaque création. Mon approche théâtrale m'a incité très tôt à concevoir le son en regard des autres éléments constitutifs du spectacle. Cela n'est pas né d'une idée, mais d'une expérience de conception sonore partagée avec un scénographe ${ }^{2}$; c'était aussi la résultante du devoir d'inscrire chaque jour du son dans le vivant des répétitions. Les multiples phases de conception d'un spectacle sont éparpillées dans le temps et les différents groupes au travail (scénographie, costumes, lumière, interprétation) peuvent facilement s'abstenir d'entretenir des relations mutuelles, chaque collaborateur développant sa propre réalisation solitaire en ne se référant qu'à la mise en scène. Comment donc travailler à construire l'unité sonore d'un spectacle dans 
cette dislocation? Si le metteur en scène détient à lui seul une vue d'ensemble, ce ne sera en fait qu'à l'arrivée des derniers filages que le spectacle commencera à apparaître à chacun. On peut supposer que si le travail sonore devait attendre cet instant pour pouvoir s'élaborer, ce serait à l'évidence trop tard. Chaque création rappelle ce paradoxe : une vision globale du projet serait idéalement nécessaire pour concevoir la nature du son d'un spectacle. On sait par exemple qu'en musique, la lecture de la partition donne cette vision d'ensemble avant même que soit engagé le travail de répétition. Au théâtre au contraire, la création ne peut se penser qu'au fil du processus de construction, hors de toute vision préalable du résultat. Le travail quotidien sur le son ne peut pourtant demeurer isolé, doivent être considérés ce que produisent l'ensemble des " acteurs sonores » : les paroles (en terme de timbre, de niveau et de directivité des voix), le bruit des déplacements, la nature des espaces résonants, et indirectement l'intensité lumineuse, l'énergie du jeu également. Plus largement encore, le dialogue qu'il nous est nécessaire d'engager - est-ce vraiment un dialogue ? - s'établit avec tout le corps théâtral - y compris la machinerie et ses rapports à l'acoustique des salles et des plateaux.

3 Le travail du son se pense autant dans l'élimination de sons que dans leur ajout. Ces retours en arrière, ces "repentirs" comme disent les peintres font apparaitre le questionnement de l'existence du silence. Les silences du spectacle ne sont jamais pensés par la mise en scène, ils arrivent toujours par défaut. Pourtant le concepteur du son les traque, et les silences assumés par la mise en scène ou par un acteur sont rares; nous les cherchons d'abord parce qu'ils représentent pour nous autant de lieux de placement possible pour nos sons. J'avais depuis bon nombre d'années pris la résolution de ne plus placer de son sous les textes, il ne me restait donc que ces instants silencieux comme lieu d'inscription. Mais l'action de la construction sonore est une construction en augmentation, nous mettons des années pour comprendre que retirer est aussi important qu'ajouter. La décision d'opter pour un silence ne pouvant se prendre trop tôt, comment résister à placer dans chaque arrêt, aussi petit soit-il, un élément sonore - ne serait-ce qu'en attendant? La question du silence finit toujours par se poser. Le silence est de toute façon le dernier élément à aménager pour que le spectacle trouve ses respirations. Il est l'interstice qui permet à l'auditeur d'intégrer sa localisation dans l'espace scénique et sa durée d'existence, car le silence appartient aux spectateurs, il leur permet aussi de faire retour inconsciemment en eux-mêmes, en leur mémoire, un retour essentiel à l'économie de l'écoute. Le travail théâtral se tient le plus souvent dans l'apnée de ses élocutions. Si la mise en scène pense toujours qu'un ralentissement $d u$ flux ${ }^{3}$ de la parole mène à l'endormissement du public, elle ne peut pour autant omettre de penser la place de ses respirations. J'ai toujours négocié avec la mise en scène le placement de silences entre les phrases dans des moments d'arrêt ou de déplacements des acteurs. La conception sonore doit veiller à la nature des éléments émis relativement aux mouvements des corps.

\section{Comment la conception sonore se constitue-t-elle dans l'espace des interstices?}

La réponse se situerait dans la confection d'un sonore s'élaborant comme un tissu. S'ils sont les constituants des organes eux-mêmes, les tissus sont aussi ceux qui établissent les liens entre les organes et les maintiennent en place. Le «treillage » qui les organise n'est pas serré, tout au contraire, la nécessaire souplesse est liée à ces interstices. Le creux interstitiel est une donnée aussi constitutive que le plein du tissu. Et ce tissu n'est pas 
constitué d'une trame fixe, il est conçu dans une élasticité dynamique. Plus sa tension est grande, plus l'interstice grandit. L'apnée dans laquelle est tenu le public est relative à cette tension. Il est indispensable qu'à l'énonciation succède un silence pour que le public reprenne son souffle. La salle entière respire avec les sons du théâtre et cela dépasse largement le seul cadre de l'énonciation du texte par les acteurs. Cette respiration constitutive, ce "collectif respiratoire» désigne bien l'existence d'une économie organisée entre plein et creux, dans l'essentielle souplesse du tissage sonore global du spectacle. Une telle souplesse constitue son audibilité.

5 Il est bon de rappeler dans une parenthèse que ce silence attendu sous la voix des acteurs n'a pas toujours existé. Il y a tout juste cinquante ans, le silence était encore réclamé à la force des coups d'un brigadier. Une puissante série était assénée avant que ne soient frappés les «trois coups» qui visaient à faire taire l'assemblée. Cette pratique était le vestige d'une époque plus bruyante encore. Ce silence se constitue à présent à partir du signe donné par la lumière, la mise au noir de la salle. L'apprentissage du silence structurel et sédimentaire du théâtre s'est peu à peu intégré à la culture du spectateur.

6 La difficulté de la fabrication de la maille réside dans le fait que le son est un phénomène temporel et qu'à ce titre on ne peut le travailler que dans le mouvement, c'est-à-dire dans une transformation quotidienne, tout au long des répétitions et jusqu'à la fin des représentations. Ces organes - texte, scénographie, costume - ne sont donc pas des objets observables à l'arrêt, mais dans leur existence vivante et instantanée, dans leurs mouvements, dans l'ensemble de leurs circulations (flux sonores des voix, des mouvements de plateau, voire des musiques jouées en direct). Ils doivent être vus dans une pensée dynamique de la brève existence de leur passage. Le son est un fluide, qu'il provienne des voix des acteurs, du bruit des mouvements des corps ou encore des sons issus des haut-parleurs, il doit être traité comme nature de flux, quelle que soit sa force sémantique. Le son dit d'abord l'énergie d'un mouvement, une manière d'entrer dans un futur immédiat qui s'annonce, c'est donc cet aspect qui est à considérer d'emblée. Conséquemment, pour que le son soit vu dans son mouvement, il faut faire retour sur les expériences passées. La pensée préalable du sonore peut exister comme mémoire des expériences, cependant chaque expérience est différente et l'approche qui peut être supposée de l'avenir demeurera à l'échelle de l'esquisse, des grandes lignes. La loi du plateau saura rapidement interroger la capacité d'existence "définitive» des sons proposés.

$7 \quad$ N'existe réellement qu'une vision d'un présent bref, en train d'avoir lieu, dans une zone flottante située entre l'épaisseur de la mémoire de ce qui vient d'advenir et dans le pressentiment de ce qui va arriver. Cet espace mnésique est court pour le spectateur comme pour le concepteur qui y sont pareillement soumis. Il constitue le lieu de la réponse silencieuse que chacun fournit, écoutant sa mémoire. Ce temps de silence est nécessaire pour parvenir à habiter les paroles et les actes perçus. C'est pourquoi il importe de ne pas densifier la matière sonore. Si trop d'éléments coexistent, aussi disséminés soient-ils, ils dérobent à notre perception la parole énoncée en scène. Plus encore, le silence détermine les conditions d'advenue de la parole suivante, c'est-à-dire que l'écoute de l'autre est indispensable à la possibilité d'énoncer et par conséquent à la possibilité d'écoute du public. Les spectateurs perçoivent quand les acteurs ne s'écoutent pas et "se mettent en automatisme", leur écoute mutuelle tombe immédiatement. Ce silence-là doit exister en pure perte; une perte de temps qui nous fait gagner en imaginaire. La représentation est le lieu où l'écrit - objet en attente de formulation - 
tombe sous la loi du temps. Cette loi est celle de l'incertitude de la durée à venir. Elle nous impose de nous y abandonner. C'est le lieu de perte d'un récit qui s'écoule, disperse et efface des sensations qui surgissaient à peine, c'est aussi le lieu de l'incessante perte de l'imaginaire des sons. Il s'agit de replacer le son en dehors de toute considération de sens, de tout commentaire de texte ou d'image, dans le présent de la représentation : dans son corps à corps avec le public.

\section{L'ancrage de la diffusion}

Placer des sons dans un spectacle exige d'avoir réglé au préalable les conditions d'écoute de la parole en corrigeant, même approximativement, l'acoustique du plateau voire de la salle. La clarté acoustique permet d'apprécier la localisation, de situer l'évènement qui répond à l'action, la complète ou la modifie. La machinerie théâtrale - superbe produit de l'histoire - nous offre encore un jeu de cintres voués jadis essentiellement à l'accrochage des toiles peintes. Elles furent remplacées au fur et à mesure de leur disparition par l'accroche des éclairages. Le son a cru bon de disputer ces perches à la lumière pour y accrocher ses haut-parleurs. S'il est important de faire disparaître les sources sonores de la vue, pourquoi donc les plafonner? Hors la sonorisation des orages ou le passage des avions, ou de quelques oiseaux, quelle nécessité y aurait-il de diffuser depuis le haut? On pourrait accepter l'idée d'une zone de diffusion vouée à l'abstrait musical, mais pourquoi serait-elle située dans les cieux? La musique serait-elle céleste? Il me semble au contraire qu'elle est le plus souvent assise sur des chaises. À cet éther musical répond assurément la nécessité d'enraciner le sonore des voix et des bruits.

9 Il faut à ce propos revenir sur l'idée de musicalité de la voix : la parole n'est pas musicale, elle est sonore. La placer dans ce registre, c'est oublier autant le sens qu'elle draîne que sa puissance tactile, son toucher. Elle bouleverse notre corps, parfois au-delà de l'acceptable voire du supportable. Elle le représente jusqu'à l'obscénité : parler de musicalité de la voix, c'est placer un cache sexe devant elle pour l'abstraire en la divinisant. On parlait jadis de bel organe pour la voix... C'est dire qu'il s'agissait bien de cette chair-là.

L'ancrage au sol des sons du théâtre est essentiel pour que fusionnent voix, bruits de circulation des corps et évènements sonores, scénographiques ou non. Or la démarche dominante de sonorisation, si elle se soucie d'une gestion de la qualité des niveaux sonores, se pense trop peu dans une localisation précise des zones de diffusion. Le cadre de scène, à l'avant du plateau, se trouve être le lieu dominant de provenance des sons, comme si ce seuil, cette porte d'entrée vers l'imaginaire, devait se définir dans un à-plat, plan limite de pénétration dans l'espace. Comme si la vision du dispositif scénographique pouvait prendre le relais du sonore; comme jadis où l'on jouait à la rampe et pénétrait très peu la profondeur des plateaux réservés aux images affectées par la réduction perspectiviste. Ce n'est pas une verticalité étagée dans la profondeur mais une frontalité massive qui est souvent proposée. Cette frontalité est utilisée dans les concerts de variété, elle envoie la pression sonore sur une salle qui doit se boucher les oreilles pour assister à la performance. Elle prend modèle sur la salle de concert classique : un espace unique rassemblant orchestre et spectateurs tenus face-à-face. La confusion avec le modèle musical induit que parfois la salle elle-même est investie par une couronne de hautparleurs englobant le public, comme si elle pouvait soudain devenir l'espace de jeu, sans plus de considérations scénographiques. L'espace unique réunissant salle et scène, l'une des multiples configurations possibles de la scénographie théâtrale est une configuration 
idéale pour mettre en place des points de diffusion travaillant comme autant de sources réelles. Cette disposition ponctuelle, localisée, s'oppose à une configuration ambiophonique circulaire. Des haut-parleurs sont disséminés pour que leur dispersion au plateau produise autant de sources réelles, seules aptes à dialoguer avec le mouvement des corps en scène.

11 Nous avons vu que si le sonore s'élabore comme un tissu qui peu à peu cartographie son espace, son tissage doit être suffisamment lâche pour que les interstices permettent à ce tramage de respirer. Nous les avons décrits disséminés dans le temps, permettant aux silences d'exister sur un texte trop tendu. Nous apercevons maintenant les interstices dans l'espace-plan du plateau. La dissémination des haut-parleurs dans des interstices d'une grille implantée au sol permet leur ancrage dans le « in » du théâtre, dans la réalité directe figurée par la scénographie. Si des transducteurs de surface étaient répartis sur toute la surface du plateau, toutes les zones de jeu pourraient être des lieux plus ou moins élargis de surgissement sonore. Nous obtiendrions alors une configuration d'émission hémisphérique, une sorte de renversement du principe utilisé par les microphones à zone de pression. C'est une élévation d'espaces qui deviendrait soudain possible, et avec elle, la mise en œuvre d'acoustiques virtuelles dans la droite ligne de ce que j'ai initié en 1985 en Avignon avec Herculine Barbin mis en scène par Alain Françon. C'était alors par enveloppement de l'espace unique scène/salle que je modifiais les acoustiques de l'espace dramatique. Je développai cette recherche de virtualité scénographique à l'Ensatt dans l'atelier Son/Scénographie que dirigea Célie Pauthe avec la pièce d'Ingmar Bergman Sarabande. La construction d'acoustiques virtuelles de ce système encore expérimental s'effectuait par un maillage au sol de sources sonores planes permettant de constituer des espaces acoustiques coexistants ${ }^{4}:$ les pièces aux acoustiques différentes d'un même logis s'accolaient virtuellement sur un même plateau.

\section{L'impossible captation sonore des spectacles}

12 Cette perception des raffinements acoustiques du théatre sonore est un privilège du direct, dont l'enregistrement ne peut encore hélas témoigner pour les années à venir. Si l'enregistrement des spectacles laisse entendre les silences, il ne permet pas en revanche de faire percevoir clairement les localisations. Du travail le plus raffiné des circulations et existences sonores, l'enregistrement ne garde presque rien. Et paradoxalement nous nous retrouverons dans les conditions de lecture des enregistrements des années passées où les localisations dans l'espace ne sont jamais clairement audibles. Que ça joue en scène, on en est sûr, mais où ça joue, on n'en sait pas grand-chose. Même simplement photographique, le secours de l'image est pour le moment toujours nécessaire pour imaginer les circulations théâtrales tant réelles que virtuelles. 


\section{BIBLIOGRAPHIE}

Deshays, Daniel, «L'impossible "façade". L'usage du microphone et son incidence sur la scénographie des espaces ", in J.-M. Larrue et M.-M. Mervant-Roux (dir.), Le Son du théâtre (XIXe$\mathrm{XXI}{ }^{e}$ siècles). Histoire intermédiale d'un lieu d'écoute moderne, Paris, CNRS Éditions, 2016, p. 261-274.

Saussure de, Ferdinand, Cours de linguistique générale, Paris, Payot, 1997.

\section{NOTES}

1. F. de Saussure, Cours de linguistique générale, p. 24.

2. Le scénographe Jean-Pierre Larroche, à la Péniche-Théâtre en novembre et décembre 1978, pour Naïves hirondelles de Roland Dubillard, mis en scène par Mireille Larroche.

3. Je vais un certain nombre de fois employer le mot flux. Il ne s'agira pas du flux qui qualifie les médias de flux (flux radiophonique, par exemple) mais il s'agit du flux sonore, des voix et autres sons, considérés pas tant pour leur continuité que pour leurs coupures, inflexions, hoquets, intensités, fragilité, matérialité, vitesse, suspension...

4. Voir ma contribution au colloque ARIAS/CRI-alt "Le son du théâtre ", à l'université de Montréal, en novembre 2012 : «L'impossible "façade". L'usage du microphone et son incidence sur la scénographie des espaces ».

\section{RÉSUMÉS}

La mise en scène pense toujours qu'un ralentissement du flux de la parole mène à l'endormissement $\mathrm{du}$ public. Elle ne peut néanmoins omettre de penser la place de ses respirations. La conception sonore doit veiller à la nature des éléments émis relativement aux paroles et aux mouvements des corps. Comment la conception sonore se constitue-t-elle dans l'espace des interstices, des silences d'un spectacle? La réponse se situerait dans la confection d'un sonore se concevant et s'élaborant comme un tissu suffisamment lâche pour que les interstices permettent à la trame de respirer. La difficulté de la fabrication de la « maille » réside dans le fait que le son est un phénomène temporel et qu'à ce titre on ne peut le travailler que dans le mouvement, c'est-à-dire dans une patiente transformation quotidienne, tout au long des répétitions et jusqu'à la fin des représentations.

Directing is always biased towards thinking that slowing down the flow of speech leads the audience to doze off. It cannot nevertheless do without meditating on the place of its breathings. The sonic design must check the nature of the elements emitted with regard to the bodies' speeches and movements. How is the sonic design constituted within the space of the interstices, of the silences of a performance? The answer would take place in the making of a sound conceiving itself and elaborating itself as a fabric loose enough for the interstices to let the woof 
breathe. The difficult making of the "stitch" lies in the fact that sound is a temporal phenomenon and that, consequently, it can only be wrought in the movement, that is to say in a daily, patient transformation, all along the rehearsals and until the very end of the performances.

\section{INDEX}

Mots-clés : sonore, tissus, silence, écoute, mise en scène

Keywords : sound fabric, silence, listening, direction

\section{AUTEUR}

\section{DANIEL DESHAYS}

Réalisateur sonore et professeur des universités, fondateur du département de Conception sonore de l'Ensatt (École nationale supérieure des arts et techniques du théâtre).

Parmi ses publications :

Pour une écriture du son, Paris, Klincksieck, 2006.

Entendre le cinéma, Paris, Klincksieck, 2010.

«Sound and gesture », in M. Aramaki et. al., (éd.), Sound, Music and Motion. 10th International Symposium, CMMR 2013, Marseille, Cham, Springer, 2014, p. 483-493.

« Paysage sonore? ? in C. Guiu et al. (dir.), Soundspaces. Espaces, expériences et politiques du sonore, Rennes, PUR, 2014, p. 25-30.

« Du proche au lointain. L'existence discontinue des bruits et sa représentation continue » in M.M. Mervant-Roux et G. Pisano (dir.), Art et bruit. Ligéia, n 141-144, Paris, juil.-déc. 2015, p. 88-93. 